\title{
Viscous effect at an orthotropic micropolar boundary surface
}

\author{
RAJNEESH KUMAR and PRAVEEN AILAWALIA ${ }^{\dagger}$ \\ Department of Mathematics, Kurukshetra University, Kurukshetra 136 119, India \\ 'Department of Applied Sciences, IEET, Makhnumajra, Baddi 173 205, Distt. \\ Solan, India \\ e-mail: $\{$ rajneesh_kuk, praveen_2117\}@ rediffmail.com
}

MS received 27 September 2004; revised 8 April 2005

\begin{abstract}
Steady state responses at viscous fluid/ orthotropic micropolar solid interfaces to moving point loads have been studied. An eigenvalue approach using the Fourier transform has been employed to solve the problem. The displacement, microrotation and stress components for the orthotropic micropolar solids so obtained in the physical domain are computed numerically by applying numerical inversion technique. Viscosity and anisotropy effects on normal displacement, normal force stress and tangential couple stress have been shown graphically for a particular model. Some special cases of interest have been presented.
\end{abstract}

Keywords. Steady state; viscous fluid; micropolar; orthotropic; Fourier transform.

\section{Introduction}

The classical theory of elasticity does not explain certain discrepancies that occur in the case of problems involving elastic vibrations of high frequency and short wavelength, that is, vibrations due to the generation of ultrasonic waves. The reason lies in the microstructure of the material which exerts a special influence at high frequencies and short wavelengths. In many engineering phenomena, including the response of solids, geological materials and composites, the assumptions of classical elasticity may not capture some significant features of the continuum response. An attempt is made to eliminate these discrepancies by suggesting that the transmission of interaction between two particles of a body through an elementary area lying within the material is affected not only by the action of a force vector but also by a moment (couple) vector. This leads to the existence of couple stress in elasticity. Polycrystalline materials, materials with fibrous or coarse grain structure, full in this category. The analysis of such materials requires incorporating the theories of oriented media. For this reason, micropolar theories were developed by Eringen $(1966 \mathrm{a}, \mathrm{b})$ for elastic solids and fluids. The micropolar theory is believed to be particularly useful in investigating materials consisting of bar-like molecules which exibit microrotation effects and which can support body and surface couples. 
The formulation and solution of anisotropic problems is far more difficult and cumbersome than of their isotropic counterparts. In recent years, the elastodynamic response of anisotropic continuum has received the attention of several researchers. In particular, transversly isotropic and orthotropic materials, which may not be distinguished from each other in plane strain and plane stress, have been more regularly studied. A review of literature on micropolar orthotropic continua shows that Iesan $(1973,1974)$ analysed the static problems of plane micropolar strain of a homogeneous and orthotropic elastic solid, torsion problem of homogeneous and orthotropic cylinders in the linear theory of micropolar elasticity and bending of orthotropic micropolar elastic beams by terminal couples. Nakamura et al (1984) applied finite element method for orthotropic micropolar elasticity. Recently Kumar \& Choudhary $(2002,2003,2004)$ have discussed various problems in orthotropic micropolar continua.

Garg \& Sharma (1991a) obtained the displacement and stresses at any point of a transversly isotropic medium due to strip loading. Garg \& Sharma (1991b) also discussed twodimensional deformation of a vertically inhomogeneous orthotropic multilayered elastic medium due to general surface loads. Every \& Kim (1994) calculated the displacement response function tensor for an infinite anisotropic elastic continuum subjected to a concentrated point force with Heaviside's step function time dependence, and examples of computed response function for zinc, diamond and silicon have been provided. Rubio-Gonzalez \& Mason (1999) examined the elastodynamic response of an infinite orthotropic material with finite crack under concentrated in-plane shear loads. Pan (2003a) discussed the threedimensional Green function due to an interior point force in an anisotropic half-space with general boundary conditions. Pan (2003b) also studied the three-dimensional Green function in an anisotropic elastic biomaterial with imperfect interface.

Dynamical response to moving loads is an interesting subject in various technological and geophysical circumstances and some recent investigations are concerned with this problem. For instance, it is of great interest in solid dynamics where ground motions and stresses can be produced by blast waves (surface pressure waves due to explosions), or by supersonic aircraft. This type of investigation occurs in many branches of engineering, for e.g in bridges and railways, beams subjected to pressure waves and piping systems subjected to two-phase flow. Other applications are encountered within the context of contact mechanics, like the problem of high velocity rocket sleds sliding over steel guide-rails. Kumar and coworkers (Kumar \& Gogna 1992; Kumar \& Deswal 2000, 2002; Kumar \& Ailawalia 2003) studied the steady state response to moving loads in micropolar theory of elasticity. Obrezanova \& Rabinovich (1996) discussed wave propagation from moving sources in the ocean. Obrezanova \& Rabinovich (1998) also investigated the problem of acoustic field produced by a source moving in a stratified fluid layer lying on a homogeneous fluid half-space. Das et al (2000) employed an integral transform technique to solve the elastodynamic problem of steady state propagation of two collinear Griffith cracks located in an orthotropic elastic layer of finite thickness sandwiched between two identical orthotropic half-planes.

In the present investigation we apply the eigenvalue approach following Fourier transformation to the moving load problem at viscous fluid/orthotropic micropolar solid interface. The solutions are obtained in the transformed domain and are inverted using a numerical inversion technique.

\section{Formulation and solution of the problem}

We consider a normal point load moving along the interface of viscous fluid (medium II)/ orthotropic micropolar solid (medium I). We consider a rectangular coordinate system 


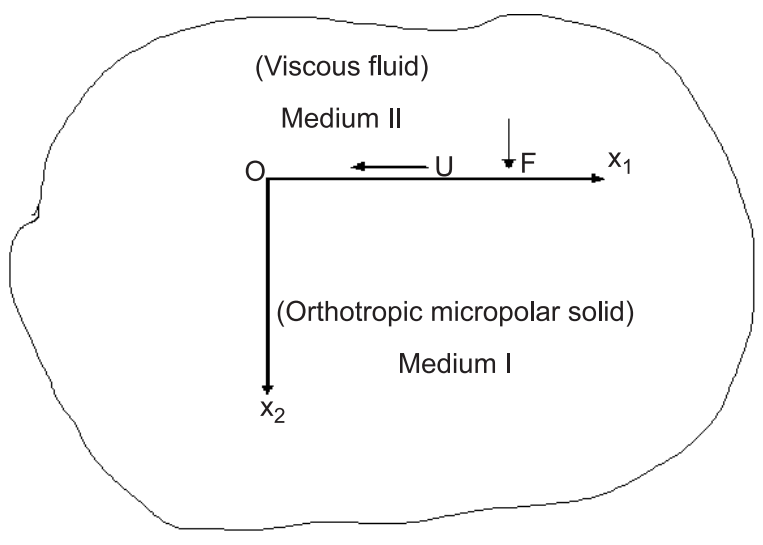

Figure 1. Moving load on interface.

$\left(x_{1}, x_{2}, x_{3}\right)$ with its origin on the surface $x_{2}=0$ and the $x_{2}$-axis pointing vertically into the medium as shown in figure 1. Let us consider a pressure pulse $P\left(x_{1}+U t\right)$ which is moving with a constant velocity in the negative $x_{1}$ direction. After the load has been moving for some time and the transient effects have died away, the displacements appear to be stationary in a coordinate system moving with the load.

If we restrict out attention to the plane deformation parallel to the $x y$ plane with displacement vector $\mathbf{u}=\left(u_{1}, u_{2}, 0\right)$ and microrotation vector $\boldsymbol{\phi}=\left(0,0, \phi_{3}\right)$, the basic equations in the dynamic theory of the plane strain of homogeneous and orthotropic micropolar solids in the absence of body forces and body couples, given by Eringen (1968), can be recalled as:

$$
\begin{aligned}
t_{k l, l} & =\rho\left(\partial^{2} u_{k} / \partial t^{2}\right), \\
m_{k 3, k}+\varepsilon_{k l 3} t_{k l} & =\rho j\left(\partial^{2} \phi_{3} / \partial t^{2}\right), \quad k=l=1,2 .
\end{aligned}
$$

The constitutive relations, given by Iesan (1973), can be written as:

$$
\begin{array}{ll}
t_{11}=A_{11} \varepsilon_{11}+A_{12} \varepsilon_{22}, & t_{12}=A_{77} \varepsilon_{12}+A_{78} \varepsilon_{21}, \quad t_{21}=A_{78} \varepsilon_{12}+A_{88} \varepsilon_{21}, \\
t_{22}=A_{12} \varepsilon_{11}+A_{22} \varepsilon_{22}, & m_{13}=B_{66} \phi_{3,1}, \quad m_{23}=B_{44} \phi_{3,2},
\end{array}
$$

where

$$
\varepsilon_{k l}=u_{l, k}+\varepsilon_{l k 3} \phi_{3}
$$

In these relations, we have used the following notations: $t_{k l}$ - components of the force stress tensor, $m_{k 3}$ - components of the couple stress tensor, $\varepsilon_{k l}$ - components of micropolar strain tensor, $u_{k}$ - components of displacement vector, $\phi_{3}$ - component of microrotation vector, $\varepsilon_{l k 3}$ - permutation symbol, $A_{11}, A_{12}, A_{77}, A_{78}, A_{88}, A_{22}, B_{44}, B_{66}$ - characteristic constants of the material, $\rho$ - the density and $j-$ the microinertia.

From (1)-(4), we obtain the field equations of the plane strain for orthotropic micropolar solids in the form:

$$
\left(A_{11} \frac{\partial^{2}}{\partial x_{1}^{2}}+A_{88} \frac{\partial^{2}}{\partial x_{2}^{2}}\right) u_{1}+\left(A_{12}+A_{78}\right) \frac{\partial^{2} u_{2}}{\partial x_{1} \partial x_{2}}-K_{1} \frac{\partial \phi_{3}}{\partial x_{2}}=\rho \frac{\partial^{2} u_{1}}{\partial t^{2}},
$$




$$
\begin{aligned}
& \left(A_{12}+A_{78}\right) \frac{\partial^{2} u_{1}}{\partial x_{1} \partial x_{2}}+\left(A_{77} \frac{\partial^{2}}{\partial x_{1}^{2}}+A_{22} \frac{\partial^{2}}{\partial x_{2}^{2}}\right) u_{2}-K_{2} \frac{\partial \phi_{3}}{\partial x_{1}}=\rho \frac{\partial^{2} u_{2}}{\partial t^{2}} \\
& \left(B_{66} \frac{\partial^{2}}{\partial x_{1}^{2}}+B_{44} \frac{\partial^{2}}{\partial x_{2}^{2}}-\aleph\right) \phi_{3}+K_{1} \frac{\partial u_{1}}{\partial x_{2}}+K_{2} \frac{\partial u_{2}}{\partial x_{1}}=\rho j \frac{\partial^{2} \phi_{3}}{\partial t^{2}}
\end{aligned}
$$

where

$$
K_{1}=A_{78}-A_{88}, \quad K_{2}=A_{77}-A_{78}, \quad \aleph=K_{2}-K_{1} .
$$

Following Fehler (1982), the equations of motion in a viscous medium (Medium II) are,

$$
\left(K^{0}+\frac{4}{3} \eta \frac{\partial}{\partial t}\right) \nabla(\nabla . \mathbf{V})-\eta \frac{\partial}{\partial t} \nabla \times(\nabla \times \mathbf{V})=\rho^{0} \frac{\partial^{2} \mathbf{V}}{\partial t^{2}},
$$

where $K^{0}$ is the bulk modulus, $\eta$ is the fluid viscosity and $\rho^{0}$ is the fluid density and $\mathbf{V}$ is the displacement in viscous medium.

The stress and displacement relations in viscous media are given by,

$$
\tau_{m n}=\left(K^{0}-\frac{2}{3} \eta \frac{\partial}{\partial t}\right) V_{k, k} \delta_{m n}+\eta \frac{\partial}{\partial t}\left(V_{m, n}+V_{n, m}\right) \cdot m=n=1,2,3 .
$$

For a two-dimensional problem, we assume

$$
\mathbf{V}=\left(V_{1}, V_{2}, 0\right)
$$

Following, Fung (1968), a Galilean transformation

$$
x_{1}^{*}=x_{1}+U t, x_{2}^{*}=x_{2}, t^{*}=t,
$$

is introduced, then the boundary conditions would be independent of $t^{*}$ and assuming the dimensionless variables defined by the expressions

$$
\begin{aligned}
& x_{1}^{\prime}=\frac{\omega^{*}}{c_{1}} x_{1}^{*}, \quad x_{2}^{\prime}=\frac{\omega^{*}}{c_{1}} x_{2}^{*}, \quad u_{1}^{\prime}=\frac{\omega^{*}}{c_{1}} u_{1}, \quad u_{2}^{\prime}=\frac{\omega^{*}}{c_{1}} u_{2}, \quad \phi_{3}^{\prime}=\frac{A_{11}}{K_{1}} \phi_{3}, \\
& t_{k l}^{\prime}=\frac{t_{k l}}{A_{11}}, \quad m_{k 3}^{\prime}=\frac{c_{1}}{B_{44} \omega^{*}} m_{k 3}, \quad V_{1}^{\prime}=\frac{\omega^{*}}{c_{1}} V_{1}, \quad V_{2}^{\prime}=\frac{\omega^{*}}{c_{1}} V_{2}, \quad \tau_{m n}^{\prime}=\frac{\tau_{m n}}{A_{11}} .
\end{aligned}
$$

where,

$$
\omega^{* 2}=\aleph / \rho j, \quad c_{1}^{2}=A_{11} / \rho,
$$

in (5)-(7) and using (12) and (13) and applying Fourier transform defined by

$$
\tilde{f}\left(\xi, x_{2}\right)=\int_{-\infty}^{\infty} f\left(x_{1}, x_{2}\right) e^{i \xi x_{1}} \mathrm{~d} x_{1},
$$

on the resulting equations (after suppressing the primes), we obtain

$$
\begin{aligned}
& D^{2} \tilde{u}_{1}=b_{11} \tilde{u}_{1}+a_{12} D \tilde{u}_{2}+a_{13} D \tilde{\phi}_{3}, \\
& D^{2} \tilde{u}_{2}=b_{22} \tilde{u}_{2}+a_{21} D \tilde{u}_{1}+b_{23} \tilde{\phi}_{3}, \\
& D^{2} \tilde{\phi}_{3}=b_{33} \tilde{\phi}_{3}+a_{31} D \tilde{u}_{1}+b_{32} \tilde{u}_{2},
\end{aligned}
$$


where

$$
\begin{aligned}
& b_{11}=\frac{\xi^{2}\left(A_{11}-\rho M_{1}^{2} c_{1}^{2}\right)}{A_{88}}, \quad b_{22}=\frac{\xi^{2}\left(A_{77}-\rho M_{1}^{2} c_{1}^{2}\right)}{A_{22}}, \quad b_{23}=-\frac{i \xi K_{1} K_{2}}{A_{11} A_{22}}, \\
& b_{32}=\frac{i \xi c_{1}^{2} K_{2} A_{11}}{\omega^{* 2} K_{1} B_{44}}, \quad b_{33}=\frac{1}{B_{44}}\left[\xi^{2}\left(B_{66}-\rho M_{1}^{2} c_{1}^{2} j\right)+\frac{\aleph c_{1}^{2}}{\omega^{* 2}}\right], \\
& a_{12}=\frac{i \xi\left(A_{12}+A_{78}\right)}{A_{88}}, \quad a_{13}=\frac{K_{1}^{2}}{A_{11} A_{88}}, \quad a_{21}=\frac{i \xi\left(A_{12}+A_{78}\right)}{A_{22}}, \\
& a_{31}=-\frac{A_{11} c_{1}^{2}}{B_{44} \omega^{* 2}}, \quad M_{1}=\frac{U}{c_{1}}, D=\frac{\mathrm{d}}{\mathrm{d} x_{2}} .
\end{aligned}
$$

Equations (16)-(18) may be written as

$$
\frac{\mathrm{d}}{\mathrm{d} x_{2}} W\left(\xi, x_{2}\right)=A(\xi) W\left(\xi, x_{2}\right)
$$

where

$$
\begin{aligned}
& W=\left(\begin{array}{c}
Z \\
D Z
\end{array}\right), \quad A=\left(\begin{array}{cc}
O & I \\
A_{1} & A_{2}
\end{array}\right), \quad Z=\left(\begin{array}{l}
\tilde{u}_{1} \\
\tilde{u}_{2} \\
\tilde{\phi}_{3}
\end{array}\right), \\
& A_{1}=\left(\begin{array}{ccc}
b_{11} & 0 & 0 \\
0 & b_{22} & b_{23} \\
0 & b_{32} & b_{33}
\end{array}\right), \quad A_{2}=\left(\begin{array}{ccc}
0 & a_{12} & a_{13} \\
a_{21} & 0 & 0 \\
a_{31} & 0 & 0
\end{array}\right) .
\end{aligned}
$$

$\mathrm{O}$ and I are respectively zero and identity matrix of order 3.

To solve (20), we assume

$$
W\left(\xi, x_{2}\right)=X(\xi) e^{q x_{2}},
$$

which leads to an eigenvalue problem. The characteristic equation corresponding to matrix $\mathrm{A}$ is given by

$$
\operatorname{det}[A-q I]=0
$$

which on expansion provides us

$$
q^{6}+\lambda_{1} q^{4}+\lambda_{2} q^{2}+\lambda_{3}=0
$$

where

$$
\begin{aligned}
\lambda_{1}= & -\left(a_{12} a_{21}+a_{13} a_{31}+b_{11}+b_{22}+b_{33}\right), \\
\lambda_{2}= & a_{12}\left(a_{21} b_{33}-b_{23} a_{31}\right)+a_{13}\left(b_{22} a_{31}-a_{21} b_{32}\right) \\
& +b_{22} b_{33}-b_{23} b_{32}+b_{11}\left(b_{22}+b_{33}\right), \\
\lambda_{3}= & b_{11}\left(b_{23} b_{32}-b_{22} b_{33}\right) .
\end{aligned}
$$


The eigenvalues of the matrix $A$ are characteristic roots of (24). The vectors $X(\xi)$ corresponding to the eigenvalues $q_{s}$ can be determined by solving the homogeneous equation

$$
[A-q I] X(\xi)=0 .
$$

The set of eigenvectors $X_{s}(\xi), s=1,2 \ldots 6$ may be obtained as

$$
X_{s}(\xi)=\left(\begin{array}{l}
X_{g_{1}}(\xi) \\
X_{g_{2}}(\xi)
\end{array}\right)
$$

where

$$
\begin{aligned}
X_{g 1}(\xi) & =\left(\begin{array}{c}
q_{g} \\
a_{g} \\
b_{g}
\end{array}\right), \quad X_{g 2}(\xi)=\left(\begin{array}{c}
q_{g}^{2} \\
a_{g} q_{g} \\
b_{g} q_{g}
\end{array}\right), \quad q=q_{g} ; g=1,2,3, \\
X_{R 1}(\xi) & =\left(\begin{array}{c}
-q_{R} \\
a_{R} \\
b_{R}
\end{array}\right), \quad X_{R 2}(\xi)=\left(\begin{array}{c}
q_{R}^{2} \\
-a_{R} q_{R} \\
-b_{R} q_{R}
\end{array}\right), \\
R & =g+3 ; q=-q_{g} ; g=1,2,3,
\end{aligned}
$$

and

$$
\begin{aligned}
a_{g} & =\frac{b_{11} b_{23}-q_{g}^{2}\left(b_{23}+a_{21} a_{13}\right)}{\nabla_{g}}, \\
b_{g} & =\frac{q_{g}^{2} a_{31}+a_{g} b_{32}}{q_{g}^{2}-b_{33}}, \\
\nabla_{g} & =q_{g}^{2} a_{13}+a_{12} b_{23}-b_{22} a_{13} .
\end{aligned}
$$

The solution of (22) is given by

$$
W\left(\xi, x_{2}\right)=\sum_{s=1}^{3}\left[D_{s} X_{s}(\xi) \exp \left(q_{s} x_{2}\right)+D_{s+3} X_{s+3}(\xi) \exp \left(-q_{s} x_{2}\right)\right] .
$$

The transformed displacements and microrotation satisfying the radiation conditions for $x_{2} \geq$ 0 (medium I) are given by,

$$
\begin{aligned}
& \tilde{u}_{1}=-q_{1} D_{4} \exp \left(-q_{1} x_{2}\right)-q_{2} D_{5} \exp \left(-q_{2} x_{2}\right)-q_{3} D_{6} \exp \left(-q_{3} x_{2}\right), \\
& \tilde{u}_{2}=a_{1} D_{4} \exp \left(-q_{1} x_{2}\right)+a_{2} D_{5} \exp \left(-q_{2} x_{2}\right)+a_{3} D_{6} \exp \left(-q_{3} x_{2}\right) \\
& \tilde{\phi}_{3}=b_{1} D_{4} \exp \left(-q_{1} x_{2}\right)+b_{2} D_{5} \exp \left(-q_{2} x_{2}\right)+b_{3} D_{6} \exp \left(-q_{3} x_{2}\right)
\end{aligned}
$$

Adopting the same approach, the displacement components satisfying the radiation conditions for $x_{2} \leq 0$ (medium II) are given by,

$$
\begin{aligned}
& \tilde{V}_{1}=q_{4} A_{1} \exp \left(q_{4} x_{2}\right)+q_{5} A_{2} \exp \left(q_{5} x_{2}\right), \\
& \tilde{V}_{2}=d_{4} A_{1} \exp \left(q_{4} x_{2}\right)+d_{5} A_{2} \exp \left(q_{5} x_{2}\right) .
\end{aligned}
$$


where

$$
\begin{aligned}
& d_{v}=\frac{q_{v}^{2}-d_{11}}{d_{12}}, \quad d_{11}=\frac{\xi c_{1}}{i \eta U \omega^{*}}\left[K^{0}-\frac{4}{3 c_{1}} i \xi \eta U \omega^{*}-\rho^{0} U^{2}\right] ; \quad v=4,5, \\
& d_{12}=\frac{c_{1}}{\eta U \omega^{*}}\left[K^{0}-\frac{1}{3 c_{1}} i \xi \eta U \omega^{*}\right], \quad d_{21}=-\frac{3 \xi^{2} U\left(\rho^{0} U c_{1}+i \xi \eta \omega^{*}\right)}{3 K^{0}-4 i \xi \eta U \omega^{*}}, \\
& d_{22}=\frac{i \xi\left(3 K^{0} c_{1}-i \xi \eta U \omega^{*}\right)}{3 K^{0} c_{1}-4 i \xi \eta U \omega^{*}}, \quad q_{4,5}^{2}=\frac{-\lambda_{4} \pm\left(\lambda_{4}^{2}-4 \lambda_{5}\right)^{1 / 2}}{2}, \\
& \lambda_{4}=-\left(d_{12} d_{22}+d_{11}+d_{21}\right), \quad \lambda_{5}=d_{11} d_{21} .
\end{aligned}
$$

\section{Boundary conditions}

For a concentrated point force, we take $P\left(x_{1}+U t\right)=F \delta\left(x_{1}^{*}\right)$, where $\delta\left(x_{1}^{*}\right)$ is the Dirac delta function and $F$ is the magnitude of force applied, therefore in moving coordinates the boundary conditions at the free surface $x_{2}=0$ are,

$$
t_{22}=\tau_{22}-F \delta\left(x^{*}\right), \quad t_{21}=\tau_{21}, \quad m_{23}=0, \quad u_{1}=V_{1}, \quad u_{2}=V_{2} .
$$

Applying the Fourier transform defined by (15) on the boundary conditions (35) and using (3), (10)-(13) and (32)-(33), we obtain the expressions for displacement components, microrotation, force stress and couple stress for orthotropic micropolar solid as,

$$
\begin{aligned}
\tilde{u}_{1}= & (F / \Delta)\left[q_{1} \Delta_{1}^{\prime} \exp \left(-q_{1} x_{2}\right)-q_{2} \Delta_{2}^{\prime} \exp \left(-q_{2} x_{2}\right)+q_{3} \Delta_{3}^{\prime} \exp \left(-q_{3} x_{2}\right)\right] \\
\tilde{u}_{2}= & -(F / \Delta)\left[a_{1} \Delta_{1}^{\prime} \exp \left(-q_{1} x_{2}\right)-a_{2} \Delta_{2}^{\prime} \exp \left(-q_{2} x_{2}\right)+a_{3} \Delta_{3}^{\prime} \exp \left(-q_{3} x_{2}\right)\right] \\
\tilde{\phi}_{3}= & -(F / \Delta)\left[b_{1} \Delta_{1}^{\prime} \exp \left(-q_{1} x_{2}\right)-b_{2} \Delta_{2}^{\prime} \exp \left(-q_{2} x_{2}\right)+b_{3} \Delta_{3}^{\prime} \exp \left(-q_{3} x_{2}\right)\right] \\
\tilde{t}_{21}= & -(F / \Delta)\left[v_{1} \Delta_{1}^{\prime} \exp \left(-q_{1} x_{2}\right)-v_{2} \Delta_{2}^{\prime} \exp \left(-q_{2} x_{2}\right)+v_{3} \Delta_{3}^{\prime} \exp \left(-q_{3} x_{2}\right)\right] \\
\tilde{t}_{22}= & -(F / \Delta)\left[r_{1} \Delta_{1}^{\prime} \exp \left(-q_{1} x_{2}\right)-r_{2} \Delta_{2}^{\prime} \exp \left(-q_{2} x_{2}\right)+r_{3} \Delta_{3}^{\prime} \exp \left(-q_{3} x_{2}\right)\right] \\
\tilde{m}_{23}= & \left(F K_{1} / A_{11} \Delta\right)\left[b_{1} q_{1} \Delta_{1}^{\prime} \exp \left(-q_{1} x_{2}\right)-b_{2} q_{2} \Delta_{2}^{\prime} \exp \left(-q_{2} x_{2}\right)\right. \\
& \left.+b_{3} q_{3} \Delta_{3}^{\prime} \exp \left(-q_{3} x_{2}\right)\right]
\end{aligned}
$$

where

$$
\begin{aligned}
& \Delta=\sum_{n=1}^{3}(-1)^{n+1} b_{n} q_{n} D_{n}, \quad D_{m}=\sum_{i=1}^{3}(-1)^{i+1} f_{i} g_{i}^{m}+\sum_{n=4}^{6}(-1)^{n} f_{n}^{m} g_{n}, \\
& f_{1}=d_{4} q_{5}-d_{5} q_{4}, \quad f_{2}=w_{2} d_{4}-w_{1} d_{5}, \quad f_{3}=w_{1} q_{5}-w_{2} q_{4}, \\
& g_{4}=w_{2} s_{1}-w_{1} s_{2}, \quad g_{5}=s_{1} q_{5}-s_{2} q_{4}, \quad g_{6}=s_{1} d_{5}-s_{2} d_{4},
\end{aligned}
$$




$$
\begin{aligned}
g_{1}^{m} & =r_{\sigma} v_{\varsigma}-r_{\varsigma} v_{\sigma}, \quad g_{2}^{m}=r_{\sigma} q_{\varsigma}-r_{\varsigma} q_{\sigma}, \quad g_{3}^{m}=r_{\sigma} a_{\varsigma}-r_{\varsigma} a_{\sigma}, \\
f_{4}^{m} & =q_{\sigma} a_{\varsigma}-a_{\sigma} q_{\varsigma}, \quad f_{5}^{m}=v_{\sigma} a_{\varsigma}-a_{\sigma} v_{\varsigma}, \quad f_{6}^{m}=q_{\sigma} v_{\varsigma}-v_{\sigma} q_{\varsigma}, \\
\Delta_{m}^{\prime} & =f_{1}\left(v_{\sigma} b_{\varsigma} q_{\varsigma}-v_{\varsigma} b_{\sigma} q_{\sigma}\right)-f_{2} q_{\sigma} q_{\varsigma}\left(b_{\sigma}-b_{\varsigma}\right)+f_{3}\left(a_{\sigma} b_{\varsigma} q_{\varsigma}-a_{\varsigma} b_{\sigma} q_{\sigma}\right), \\
r_{m} & =\left(q_{m} / A_{11}\right)\left(i \xi A_{12}-a_{m} A_{22}\right), \\
s_{\Theta} & =\left(q_{\Theta} / A_{11}\right)\left[K^{0}\left(i \xi+d_{\Theta}\right)+\left(2 / 3 c_{1}\right) i \xi \eta U \omega^{*}\left(i \xi-2 d_{\Theta}\right)\right], \\
v_{m} & =-i \xi a_{m} A_{78}+q_{m}^{2} A_{88}+\left(K_{1} / A_{11}\right)\left(A_{88}-A_{78}\right) b_{m}, \\
w_{\Theta} & =\left(i \xi \eta U \omega^{*} / c_{1}\right)\left(q_{\Theta}^{2}-i \xi d_{\Theta}\right), \\
m & =1,2,3 ; \quad \Theta=1,2 ; \quad \sigma=2,1,1 ; \quad \varsigma=3,3,2 .
\end{aligned}
$$

\section{Particular cases}

\section{(a) Micropolar transversly isotropic materials}

This type of medium has only one axis of symmetry and in this case the elastic constants are given by

$$
A_{11}=A_{22}, \quad A_{12}, \quad A_{77}=A_{88}, \quad A_{78}, \quad B_{44}=B_{66} .
$$

Using these values in (36)-(41), we obtain the corresponding expressions in case of micropolar transversly isotropic material.

(b) Micropolar cubic crystal

The elastic constants in case of micropolar cubic crystal are given by

$$
A_{11}=A_{22}, \quad A_{12}, \quad A_{77}=A_{78}=A_{88}, \quad B_{44}=B_{66} .
$$

Using these elastic constants in (36)-(41) yield the corresponding expressions in case of micropolar cubic crystal.

(c) Micropolar isotropic material

Taking

$$
\begin{aligned}
& A_{11}=A_{22}=\lambda+2 \mu+K, \quad A_{77}=A_{88}=\mu+K, \quad A_{12}=\lambda, \quad A_{78}=\mu, \\
& B_{44}=B_{66}=\gamma,
\end{aligned}
$$

with

$$
-K_{1}=K_{2}=\aleph / 2=K,
$$

we obtain the corresponding expressions for the micropolar isotropic medium. These results tally with the one obtained for a micropolar isotropic medium.

(d) Inviscous fluid

If the viscosity of the fluid is zero, or assumed to be zero, then the fluid cannot support shear. The shear stress in the solid must vanish at the point of contact with the fluid. The solid can slip parallel to the fluid/solid interface so there is no condition on the displacement $\mathbf{V}$. The boundary value problem reduces to four equations with four unknowns when viscosity is zero. 


\section{Inversion of the transform}

To obtain the solution of the problem in the physical domain, we must invert the transform in (36)-(41). These expressions are functions of $x_{2}$ and the parameter of Fourier transform $\xi$, hence are of the form $\tilde{f}\left(\xi, x_{2}\right)$. To get the function $f\left(x_{1}, x_{2}\right)$ in the physical domain we invert the Fourier transform using,

$$
\begin{aligned}
f\left(x_{1}, x_{2}\right) & =\frac{1}{2 \pi} \int_{-\infty}^{\infty} \tilde{f}\left(\xi, x_{2}\right) e^{-i \xi x_{1}} \mathrm{~d} \xi \\
& =\frac{1}{2 \pi} \int_{-\infty}^{\infty}\left[\operatorname{Cos}\left(\xi x_{1}\right) f_{e}-i \operatorname{Sin}\left(\xi x_{1}\right) f_{o}\right] \mathrm{d} \xi .
\end{aligned}
$$

where $f_{e}$ and $f_{o}$ are respectively even and odd parts of the function $\tilde{f}\left(\xi, x_{2}\right)$. The method for evaluating this integral is described by Press et al (1986) which involves the use of Rhomberg's integration with adaptive step size. This also uses the results from successive refinements of the extended trapezoidal rule followed by extrapolation of the results to the limit when the step size tends to zero.

\section{Numerical results and discussions}

For numerical computations, we take the following values of relevant parameters for orthotropic micropolar solid as,

$$
\begin{aligned}
& A_{11}=13.97 \mathrm{~Pa}, \quad A_{77}=3.0 \mathrm{~Pa}, \quad A_{88}=3.2 \mathrm{~Pa}, \\
& A_{22}=13.75 \mathrm{~Pa}, \quad A_{12}=8.13 \mathrm{~Pa}, \quad A_{78}=2.2 \mathrm{~Pa}, \\
& B_{44}=5.6 \times 10^{2} \mathrm{~N}, \quad B_{66}=5.7 \times 10^{2} \mathrm{~N} .
\end{aligned}
$$

For comparison with micropolar isotropic solid, following Gauthier (1982), we take the following values of relevant parameters for the case of aluminium epoxy composite as,

$$
\begin{aligned}
& \rho=2.19 \times 10^{3} \mathrm{~kg} / \mathrm{m}^{3}, \quad \lambda=7.59 \mathrm{~Pa}, \quad \mu=1.89 \mathrm{~Pa}, \\
& K=1.49 \times 10^{-2} \mathrm{~Pa}, \quad \gamma=2.68 \times 10^{3} \mathrm{~N}, \quad j=0.196 \times 10^{-6} \mathrm{~m}^{2} .
\end{aligned}
$$

The values of physical constants for a few fluids are given in table 1 (White 1994).

Table 1. Values of physical constants for various fluids.

\begin{tabular}{lccc}
\hline Fluid & $\begin{array}{c}\text { Density, } \rho^{0}\left(\mathrm{~kg} / \mathrm{m}^{3}\right) \\
\left(\times 10^{3}\right)\end{array}$ & $\begin{array}{c}\text { Viscosity, } \eta(\mathrm{kg} / \mathrm{ms}) \\
\left(\times 10^{-7}\right)\end{array}$ & $\begin{array}{c}\text { Bulk modulus, } K^{0}\left(\mathrm{~N} / \mathrm{m}^{2}\right) \\
\left(\times 10^{9}\right)\end{array}$ \\
\hline Gasoline & 0.68 & 0.292 & 0.958 \\
Seawater & 1.025 & 1.07 & 2.33 \\
Kerosene & 0.804 & 1.92 & 1.6 \\
\hline
\end{tabular}




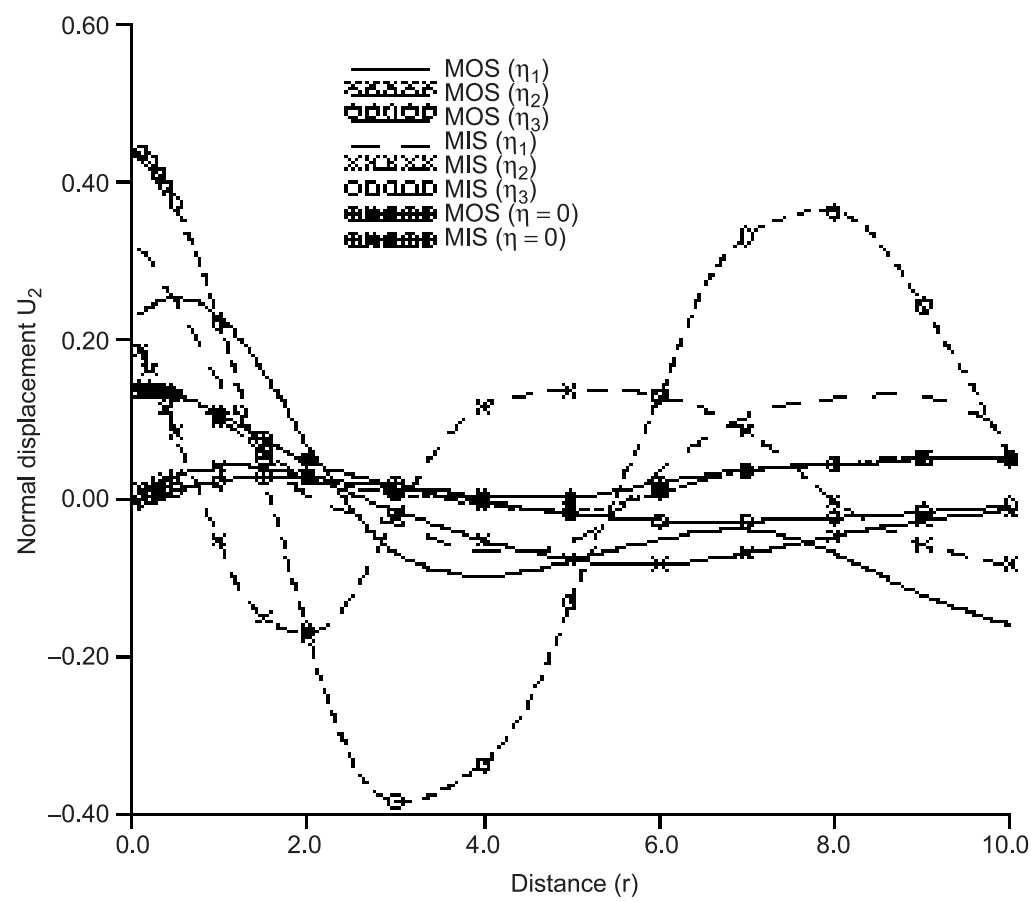

Figure 2. Variation of normal displacement $U_{2}\left(=u_{2} / F\right)$ with distance $x$.

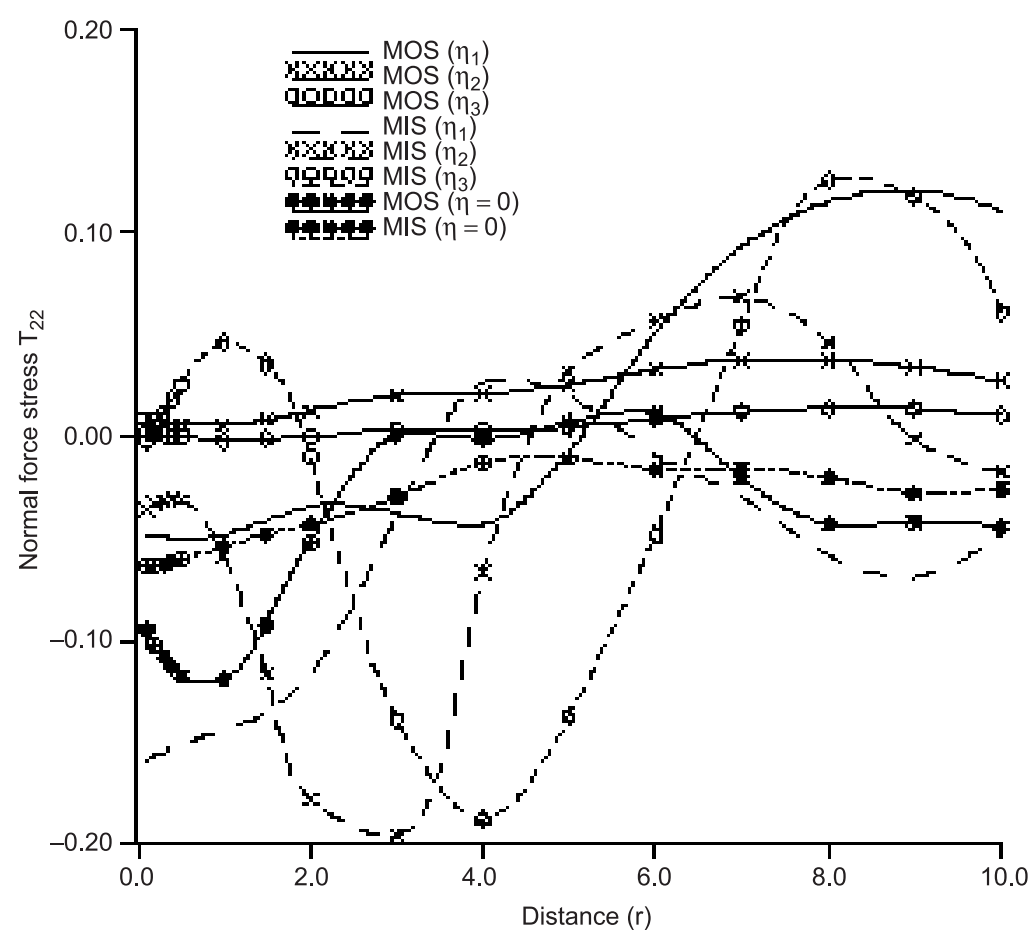

Figure 3. Variation of normal force stress $T_{22}\left(=t_{22} / F\right)$ with distance $x$. 


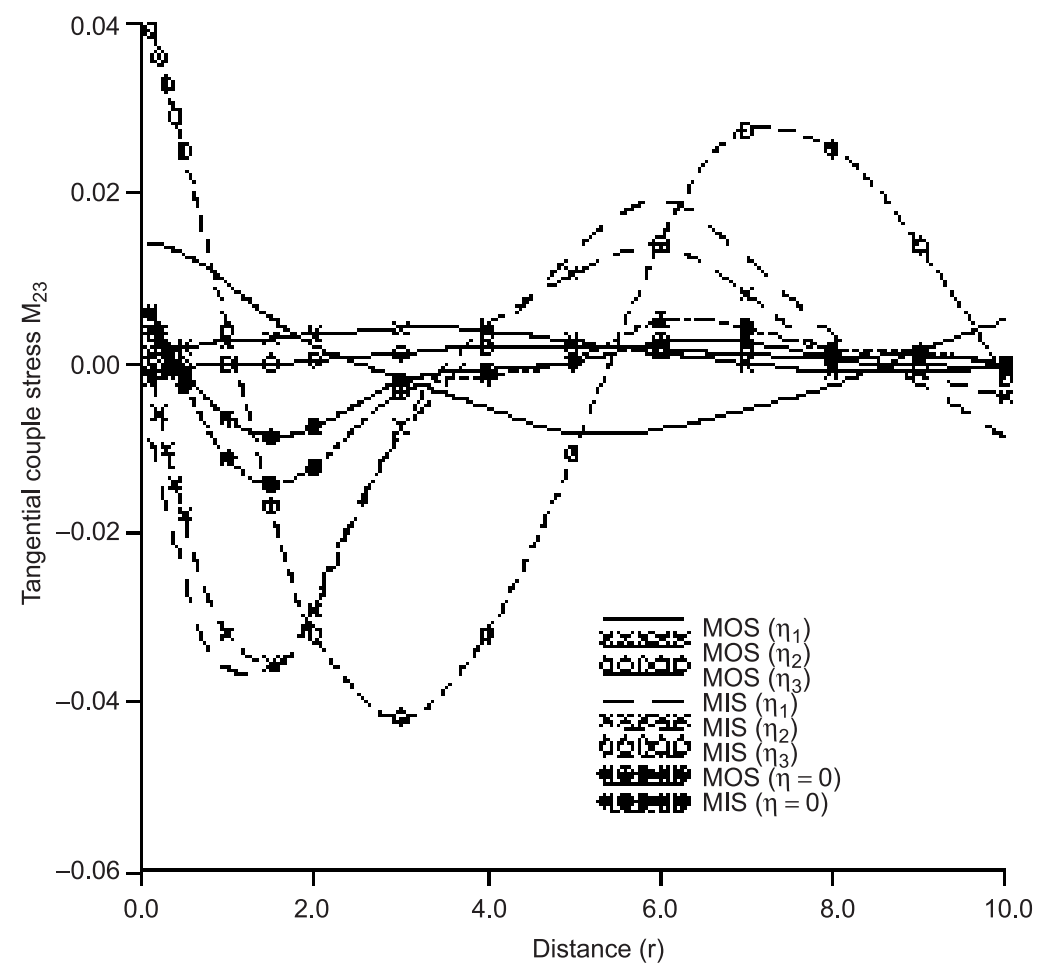

Figure 4. Variation of tangential couple stress $M_{23}\left(=m_{23} / F\right)$ with distance $x$.

The values of normal displacement $U_{2}=\left(u_{2} / F\right)$, normal force stress $T_{22}=\left(t_{22} / F\right)$ and tangential couple stress $M_{23}=\left(m_{23} / F\right)$ for an orthotropic micropolar solid (MOS) and isotropic micropolar solid (MIS) have been studied for $M_{1}<1$ and the variations of these components with distance $x$ have been shown in figures 2-4. Comparison between an orthotropic solid and an isotropic solid is also shown. The computations are carried out for $x_{2}=1 \cdot 0$ in the range $0 \leq x_{1} \leq 10 \cdot 0$.

\section{Discussions for various cases}

The values of normal displacement for MOS are lower compared to the values for MIS irrespective of the viscosity of the fluid. While the variations of normal displacement for MOS decrease with increase in the viscosity of fluid, these variations are opposite in nature for MIS. These variations of normal displacement for both MOS and MIS have been shown in figure 2 .

The values of normal force stress and tangential couple stress for MIS, near the point of application of source, increase with increase in viscosity of fluid over the surface of solid. The variations which are oscillatory in nature also increase with viscosity of fluid for MIS. The values of these quantities for MOS lie in a very short range as compared to the values for MIS. Furthermore, the range in which the values of these quantities lie for MOS decreases as the viscosity of fluid increases. The variations of normal force stress and tangential couple stress for both the solids are shown in figures 3 and 4 respectively. 


\section{Conclusion}

Anisotropy and viscous effects are quite significant in the study of deformation of a body. The values of all the quantities for MOS lie in a short range as compared to the values for MIS. Also the values of normal force stress and tangential couple stress, very close to the point of application of source, increase with increase in the viscosity of fluid over the surface of solid.

\section{References}

Das S, Patra B, Debnath L 2000 Stress intensity factors around two co-planar Griffith cracks in an orthotropic layer sandwiched between two identical orthotropic half-planes. Int. J. Eng. Sci. 38: $121-133$

Eringen A C 1966a Linear theory of micropolar elasticity. J. Math. Mech. 15: 909-923

Eringen A C 1966b Theory of micropolar fluids. J. Math. Mech. 16: 1-18

Eringen A C 1968 Theory of micropolar elasticity in fracture (New York: Academic Press) vol. 2, pp 621-729

Every A G, Kim K Y 1994 Time domain dynamic response functions of elastically anisotropic solids. J. Acous. Soc. Am. 95: 2505-2516

Fehler M 1982 Interactions of seismic waves with a viscous liquid layer. Bull. Seism. Soc. Am. 72: $55-72$

Fung Y C 1968 Foundations of solid mechanics (New Delhi: Prentice Hall)

Gauthier R D 1982 Experimental investigations on micropolar media. In Mechanics of micropolar media (eds) O Brulin, R K T Hsieh (Singapore: World Scientific)

Garg N R, Sharma R K 1991a Displacements and stresses at any point of a transversly isotropic multilayered half-space due to strip loading. Indian J. Pure Appl. Math. 22: 859-877

Garg NR, Sharma R K 1991b Two-dimensional deformation of a vertically inhomogeneous orthotropic multilayered medium due to general surface loads. Indian J. Pure Appl. Math. 22: 1035-1050

Iesan D 1973 The plane micropolar strain of orthotropic elastic solids. Arch. Mech. 25: 547-561

Iesan D 1974a Torsion of anisotropic elastic cylinders. Z. Angew. Math. Mech. 54: 773-779

Iesan D 1974b Bending of orthotropic micropolar elastic beams by terminal couples. An. Stiint. Uni. AI. I. Cuza. Iasi 20: 411-418

Kumar R, Ailawalia P 2003 Moving load response at thermal conducting fluid and micropolar solid interface. Int. J. Appl. Mech. Eng. 8: 621-636

Kumar R, Choudhary S 2002a Influence of Green's function for orthotropic micropolar continua. Arch. Mech. 54: 185-198

Kumar R, Choudhary S 2002b Dynamical behavior of orthotropic micropolar elastic medium. J. Vibr. Contr. 8: 1053-1069

Kumar R, Choudhary S 2002c Mechanical sources in orthotropic micropolar continua. Proc. Indian Acad. Sci. (Earth Plant. Sci.) 111: 133-141

Kumar R, Choudhary S 2003 Response of orthotropic microploar elastic medium due to various sources. Meccanica 38: 349-368

Kumar R, Choudhary S 2004 Response of orthotropic micropolar elastic medium due to time harmonic sources. Sādhanā 29: 83-92

Kumar R, Deswal S 2000 Steady state response of a micropolar generalized thermoelastic half space to the moving mechanical/thermal loads. Proc. Indian Acad. Sci. (Math. Sci.) 110: 449-465

Kumar R, Deswal S 2002 Steady state response to moving loads in a micropolar generalized thermoelastic half space without energy dissipation. Ganita 53: 23-42

Kumar R, Gogna M L 1992 Steady state response to moving loads in micropolar elastic medium with stretch. Int. J. Eng. Sci. 30: 811-820 
Nakamura S, Benedict R, Lakes R 1984 Finite element method for orthotropic micropolar elasticity. Int. J. Eng. Sci. 22: 319-330

Obrezanova O A, Rabinovich V S 1996 Wave propagation from moving sources in the ocean. Proc. 3rd ECCOMAS. Comput. Fluid Dynamics Conf. Paris, pp 754-759

Obrezanova O A, Rabinovich V S 1998 Acoustic field generated by moving sources in stratified wave guides. Wave Motion 27: 155-167

Pan E 2003a Three-dimensional Green's function in an anisotropic half-space with general boundary conditions. J. Appl. Mech., Trans. ASME. 70: 101-110

Pan E 2003b Three-dimensional Green's function in an anisotropic elastic bimaterials with imperfect interfaces. J. Appl. Mech., Trans. ASME. 70: 180-190

Press W H, Teukolsky S A, Vellerling W T, Flannery B P 1986 Numerical recipes (Cambridge: University Press)

Rubio-Gonzalez C, Mason J J 1999 Response of finite cracks in orthotropic materials due to concentrated impact shear loads. J. Appl. Mech. Trans. ASME. 66: 485-491

White F M 1994 Fluid mechanics (New York: McGraw Hill) 